\title{
Isometries of the spaces of self-adjoint traceless operators
}

\author{
Gergő Nagy \\ MTA-DE "Lendület" Functional Analysis Research Group, Institute of Mathematics \\ University of Debrecen \\ H-4010 Debrecen, P.O. Box 12, Hungary
}

\begin{abstract}
In this paper we describe the structure of all isometries of the space of self-adjoint traceless operators on a finite dimensional Hilbert space under the metrics coming from the operator norm or the Schatten norms. We prove that up to a translation and multiplication by -1 , they are unitary or antiunitary similarity transformations.
\end{abstract}

Keywords: Isometries, self-adjoint traceless operators, operator norm, Schatten norms 2010 MSC: Primary: 15A86, Secondary: 47B49.

Throughout this paper $\mathcal{H}$ denotes a finite dimensional complex Hilbert space. Recently, in [7] Hatori has determined the structure of isometries of the special unitary group $\mathcal{S U}(\mathcal{H})$ on $\mathcal{H}$. This group - which is the set of unitary operators on $\mathcal{H}$ with determinant 1 - is strongly connected to the linear space $\mathcal{S}_{0}(\mathcal{H})$ of all self-adjoint operators on $\mathcal{H}$ whose trace is 0 . In fact, it is a folk result that for an operator $W$ on $\mathcal{H}$ one has $W \in \mathcal{S U}(\mathcal{H})$ if and only if $W=e^{i A}$, where $A \in \mathcal{S}_{0}(\mathcal{H})$ is an element. So, roughly speaking we can say that $\mathcal{S}_{0}(\mathcal{H})$ consists of the arguments of operators in $\mathcal{S U}(\mathcal{H})$. Moreover, it is also well-known that the Lie algebra of the Lie group $\mathcal{S U}(\mathcal{H})$ consists of the elements of $\mathcal{S}_{0}(\mathcal{H})$ multiplied by i. In the light of these facts, it is not surprising that there is a connection between the isometries of $\mathcal{S}_{0}(\mathcal{H})$ and of $\mathcal{S U}(\mathcal{H})$. Indeed, as [7, Lemma 2.1.] shows, any isometry of $\mathcal{S U}(\mathcal{H})$ with respect to the metric coming from the operator norm gives rise to a surjective linear isometry of $\mathcal{S}_{0}(\mathcal{H})$. The proof of [7, Theorem 1.1.] on the structure of such maps of $\mathcal{S U}(\mathcal{H})$ is based on several lemmas and involves long computations which were necessary, since, as far as we are concerned there is no structural result for the isometries of $\mathcal{S}_{0}(\mathcal{H})$ in the literature. Motivated mainly by the latter facts, in this paper we describe the general form of those transformations. Having a closer look at the mentioned proof and at Theorem below, it turns out that applying that result, the verification of [7, Theorem 1.1.] can be greatly shortened.

The result in this paper is classified into the broad field of isometries of normed spaces which has a vast literature extending to several parts of mathematics. For a collection of results in the latter area the reader can consult, e.g. the books [5, 6]. A particular subarea of this branch of mathematics is that of the isometries on spaces of bounded linear operators which is investigated by many authors. We remark that in the rest of the present paragraph by an isometry we mean not just an arbitrary distance preserving map, but a transformation leaving the metric induced by the operator norm invariant. The description of surjective linear isometries of classical normed spaces of operators is known. In cases of the algebra of bounded linear operators on $\mathcal{H}$ and the

Email address: nagyg@science.unideb.hu (Gergő Nagy) 
set $\mathcal{S}(\mathcal{H})$ of self-adjoint operators on $\mathcal{H}$, they can be determined using the results in [8] and [9], respectively. The bijective linear isometries of $\mathcal{S}(\mathcal{H})$ are, up to multiplication by -1 , unitary or antiunitary similarity transformations. We remark that this fact can be applied to determine all isometries of $\mathcal{S}(\mathcal{H})$. Indeed, the argument given in the first paragraph of the proof of Theorem below shows that, up to a translation, each isometry $\phi$ of $\mathcal{S}(\mathcal{H})$ is surjective and linear. Now it follows that $\phi$ is of the form $\phi(A)=\tau U A U^{*}+X(A \in \mathcal{S}(\mathcal{H}))$, where $U$ is a unitary or an antiunitary operator on $\mathcal{H}, X \in \mathcal{S}(\mathcal{H})$ is an operator and $\tau \in\{-1,1\}$ is a number. The main aim of this paper is to show that an analogous statement holds also for the isometries of $\mathcal{S}_{0}(\mathcal{H})$.

In what follows, we collect some basic facts about self-adjoint traceless operators on $\mathcal{H}$. In the case $\mathcal{H}=\mathbb{C}^{2}$, they play an important role in the mathematical foundations of quantum mechanics. In fact, the so-called spin operators all belong to $\mathcal{S}_{0}\left(\mathbb{C}^{2}\right)$ and the well-known Pauli matrices (considered as operators on $\mathbb{C}^{2}$ ) form a basis in it. It is worth mentioning that $\mathcal{S}_{0}(\mathcal{H}) \subset \mathcal{S}(\mathcal{H})$ has codimension 1. Indeed, $\mathcal{S}(\mathcal{H})$ is a Hilbert space endowed with the so-called Hilbert-Schmidt inner product and the orthogonal complement of $\mathcal{S}_{0}(\mathcal{H})$ is the set of all scalar operators in $\mathcal{S}(\mathcal{H})$. It is a well-known result that complex matrices with trace 0 are exactly the commutators of pairs of matrices. An analogous statement holds also for the elements of $\mathcal{S}_{0}(\mathcal{H})$, namely they are precisely those operators $A$ on $\mathcal{H}$ for which $A$ is the product of i and the commutator of a pair of operators in $\mathcal{S}(\mathcal{H})$.

Turning to our result, under some additional assumptions we give the structure of isometries of $\mathcal{S}_{0}(\mathcal{H})$ relative to the metric $d_{\infty}$ induced by the operator norm $\|.\|_{\infty}$ or with respect to the distances coming from the Schatten norms. The class of the latter functions contains the trace norm $\|\cdot\|_{1}$ and the Hilbert-Schmidt norm $\|.\|_{2}$ which comes from the Hilbert-Schmidt inner product. As for $\|\cdot\|_{1}$ and $\|.\|_{2}$, we remark that they are frequently used in several areas of mathematics. The Schatten norms can be defined as follows. Let $p \geq 1$ be a real number and $A$ be a linear operator on $\mathcal{H}$. Moreover we denote by tr the trace functional. The Schatten $p$-norm $\|A\|_{p}$ of $A$ is defined by

$$
\|A\|_{p}=\left(\operatorname{tr}\left(A^{*} A\right)^{\frac{p}{2}}\right)^{\frac{1}{p}} .
$$

In the proof of the below theorem, we will implicitly use the following formulas. One has

$$
\|A\|_{p}=\left(\sum_{i=1}^{n}\left|\lambda_{i}\right|^{p}\right)^{\frac{1}{p}},\|A\|_{\infty}=\max \left\{\left|\lambda_{i}\right|: i=1, \ldots, n\right\}
$$

for any self-adjoint operator $A$ on the $n$-dimensional space $\mathcal{H}$ with eigenvalues $\lambda_{1} \leq \ldots \leq \lambda_{n}$. In the rest of the paper $d_{p}$ stands for the metric coming from the $p$-norm. Now we are in a position to formulate the result concerning isometries of $\mathcal{S}_{0}(\mathcal{H})$ with respect to $d_{p}(1 \leq p \leq \infty)$.

Theorem. Let $2 \neq p \geq 1$ be an extended real number and $\phi: \mathcal{S}_{0}(\mathcal{H}) \rightarrow \mathcal{S}_{0}(\mathcal{H})$ be an isometry with respect to $d_{p}$. If $p=\infty$, then there exist a unitary or an antiunitary operator $U$ on $\mathcal{H}$ and an operator $X \in \mathcal{S}_{0}(\mathcal{H})$ such that $\phi$ can be written in the form

$$
\phi(A)=U A U^{*}+X \quad\left(A \in \mathcal{S}_{0}(\mathcal{H})\right)
$$

or in the form

$$
\phi(A)=-U A U^{*}+X \quad\left(A \in \mathcal{S}_{0}(\mathcal{H})\right) .
$$

Moreover, this conclusion holds true also in the case where $p<\infty$ and $\operatorname{dim} \mathcal{H} \neq 3$. 
Before presenting the proof of the above result let us make a few remarks. Let $2 \neq p \geq 1$ be an extended real number and assume that $p=\infty$, or $p<\infty$ and $\operatorname{dim} \mathcal{H} \neq 3$. Consider $\mathcal{S}(\mathcal{H})$ as a metric space endowed with $d_{p}$. As for the converse of Theorem, one can check that the transformations of one of the forms appearing in it are isometries of $\mathcal{S}_{0}(\mathcal{H})$. The mentioned result shows that any isometry of $\mathcal{S}_{0}(\mathcal{H})$ can be extended to that of $\mathcal{S}(\mathcal{H})$. Finally, we mention that using Theorem, we can determine the general form of the isometries of the set of elements in $\mathcal{S}(\mathcal{H})$ with a fixed trace. Indeed, let $a$ be a real number, $\mathcal{S}_{a}(\mathcal{H})$ be the space of all operators in $\mathcal{S}(\mathcal{H})$ of trace $a$ and $\phi$ be an isometry of $\mathcal{S}_{a}(\mathcal{H})$. Then it is clear that the map $\psi: \mathcal{S}_{0}(\mathcal{H}) \rightarrow \mathcal{S}_{0}(\mathcal{H})$ defined by

$$
\psi(A)=\phi(A+(a / \operatorname{dim} \mathcal{H}) I)-(a / \operatorname{dim} \mathcal{H}) I \quad\left(A \in \mathcal{S}_{0}(\mathcal{H})\right)
$$

is an isometry and thus by Theorem, it is of one of the forms appearing in it (in this paper $I$ denotes the identity operator). Next, transforming back to $\phi$ we get that

$$
\phi(A)=U A U^{*}+X_{1} \quad\left(A \in \mathcal{S}_{a}(\mathcal{H})\right)
$$

or

$$
\phi(A)=-U A U^{*}+X_{2} \quad\left(A \in \mathcal{S}_{a}(\mathcal{H})\right)
$$

with some operators $X_{1} \in \mathcal{S}_{0}(\mathcal{H}), X_{2} \in \mathcal{S}_{2 a}(\mathcal{H})$ and a unitary or antiunitary operator $U$. Now we present the verification of Theorem.

Proof. Since Theorem trivially holds in the case where $n \doteq \operatorname{dim} \mathcal{H}=1$, in the rest of this section we assume that $n>1$. Consider an isometry $\phi$ of the finite dimensional space $\mathcal{S}_{0}(\mathcal{H})$ with respect to $d_{p}$. Regarding the statements in Theorem, it is clear that without loss of generality, we may and do assume that $\phi(0)=0$. The following argument shows that then $\phi$ is surjective and linear (see also [4]). In fact, by the invariance of domain, it follows easily that the image rng $\phi$ of the open set $\mathcal{S}_{0}(\mathcal{H})$ under $\phi$ is such a set. On the other hand, since $\mathcal{S}_{0}(\mathcal{H})$ is also complete, so is rng $\phi$. These observations yield that $\operatorname{rng} \phi$ is a closed and open subset of $\mathcal{S}_{0}(\mathcal{H})$ and hence $\operatorname{rng} \phi=\mathcal{S}_{0}(\mathcal{H})$ which implies that $\phi$ is surjective. The Mazur-Ulam theorem tells us that any surjective isometry between real normed spaces is affine. Since $\phi(0)=0$ we obtain that $\phi$ is linear completing our argument.

In this section we denote by $\mathcal{P}_{1}(\mathcal{H})$ the set of the rank-one projections in $\mathcal{S}(\mathcal{H})$. We divide the rest of the proof into two cases.

CASE I. In this part, we assume that $p=\infty$. We are going to prove the first statement in Theorem by induction on $n$. Accordingly, first suppose that $n=2$. Then it is clear that $\phi$ preserves $\|\cdot\|_{\infty}$, i.e. for any $A \in \mathcal{S}_{0}(\mathcal{H})$ we have $\|\phi(A)\|_{\infty}=\|A\|_{\infty}$. Now consider an operator $A \in \mathcal{S}_{0}(\mathcal{H})$. Then it is straightforward to see that there exists a projection $P \in \mathcal{P}_{1}(\mathcal{H})$ and a real number $a \geq 0$ such that $A=a(2 P-I)$. This gives us $\|A\|_{\infty}=a$ yielding that $\phi$ preserves the operators of the form $2 P-I$ with some element $P \in \mathcal{P}_{1}(\mathcal{H})$. We deduce that the transformation $\psi: \mathcal{P}_{1}(\mathcal{H}) \rightarrow \mathcal{P}_{1}(\mathcal{H})$ defined by

$$
\psi(P)=\frac{1}{2}(\phi(2 P-I)+I) \quad\left(P \in \mathcal{P}_{1}(\mathcal{H})\right)
$$

is a surjective isometry of $\mathcal{P}_{1}(\mathcal{H})$. By Wigner's theorem (cf. [3, Theorem 1.1.]), such maps of the set of rank-one projections on a Hilbert space are unitary or antiunitary similarity transformations. Applying this result to $\psi$, we obtain that there is a unitary or an antiunitary operator such that $\psi$ is of the form $\psi(P)=U P U^{*}\left(P \in \mathcal{P}_{1}(\mathcal{H})\right)$. We infer that

$$
\phi(2 P-I)=U(2 P-I) U^{*} \quad\left(P \in \mathcal{P}_{1}(\mathcal{H})\right)
$$


which, by homogeneity implies that $\phi(A)=U A U^{*}\left(A \in \mathcal{S}_{0}(\mathcal{H})\right)$ and hence we get the first statement of Theorem in the case $n=2$.

Next suppose that $n>2$ and this statement holds for any complex Hilbert space with dimension $n-1$. We have seen that the isometry $\phi$ is surjective and linear, hence it maps the unit sphere $\mathcal{S}_{0}(\mathcal{H})_{1}$ of $\mathcal{S}_{0}(\mathcal{H})$ onto itself. We proceed with the characterization of distance 2 elements in $\mathcal{S}_{0}(\mathcal{H})_{1}$. Namely, for any pair $A, B \in \mathcal{S}_{0}(\mathcal{H})_{1}$ of operators we have $d_{\infty}(A, B)=2$ if and only if 1 , resp. -1 is an eigenvalue for $A$, resp. $B$ or reversely and the corresponding eigenspaces have nontrivial intersection. Let $A, B \in \mathcal{S}_{0}(\mathcal{H})_{1}$. Then using the condition $\operatorname{dim} \mathcal{H}<\infty$ and the fact that the operator norm of a self-adjoint operator coincides with its numerical radius, we infer that there is a unit vector $x_{0} \in \mathcal{H}$ such that

$$
\begin{gathered}
d_{\infty}(A, B)=\sup \{|\langle(A-B) x, x\rangle|: x \in \mathcal{H},\|x\|=1\} \\
=\left|\left\langle A x_{0}, x_{0}\right\rangle-\left\langle B x_{0}, x_{0}\right\rangle\right| .
\end{gathered}
$$

Since $\|A\|_{\infty}=\|B\|_{\infty}=1$, we see that the numerical ranges of $A$ and $B$ lie in $[-1,1]$ and then it follows that $d_{\infty}(A, B)=2$ exactly when $\left\langle A x_{0}, x_{0}\right\rangle$ is 1 and $\left\langle B x_{0}, x_{0}\right\rangle$ is -1 , or reversely. It is very easy to check that $\left\langle A x_{0}, x_{0}\right\rangle= \pm 1$ if and only if $x_{0}$ is an eigenvector of $A$ with eigenvalue \pm 1 and the same assertion holds also for $B$. Now we conclude that the desired characterization is valid. In a very similar way it can be shown that $\|A+B\|_{\infty}=2$ if and only if at least one of the numbers $-1,1$ is a common eigenvalue for $A, B$ and their corresponding eigenspaces have nonzero intersection.

We define (also in the case $n=2$ )

$$
\mathcal{D P}_{1}(\mathcal{H})=\left\{P-Q \mid P, Q \in \mathcal{P}_{1}(\mathcal{H}), P Q=0\right\} .
$$

Now assume that $n=3$. Then using an argument similar to the one in the previous paragraph it can be shown that the elements of $\mathcal{D} \mathcal{P}_{1}(\mathcal{H})$ are extreme points of the unit ball of $\mathcal{S}_{0}(\mathcal{H})$. Moreover, it is very easy to see that the elements of the complement of $\mathcal{D} \mathcal{P}_{1}(\mathcal{H})$ with respect to this ball are not such points. We deduce that the set of extreme points in question is $\mathcal{D} \mathcal{P}_{1}(\mathcal{H})$, therefore the surjective linear isometry $\phi$ leaves it invariant. It is straightforward to see that the elements of $\mathcal{S}_{0}(\mathcal{H})_{1} \backslash \mathcal{D} \mathcal{P}_{1}(\mathcal{H})$ are exactly the operators $A \in \mathcal{S}_{0}(\mathcal{H})_{1}$ with the following property.

(*) There is a scalar $\tau \in\{-1,1\}$ for which $\tau$ is a simple eigenvalue of $A$ and $-\tau \notin \sigma(A)$ (in this paper $\sigma($.$) denotes the spectrum of operators).$

Now we obtain that $\phi$ preserves the elements of $\mathcal{S}_{0}(\mathcal{H})_{1}$ with the property $\left(^{*}\right)$.

Next suppose that $n>3$. We define a relation $\sim$ on $\mathcal{S}_{0}(\mathcal{H})_{1}$ in the following way. Let $A, B \in \mathcal{S}_{0}(\mathcal{H})_{1}$ be operators. We write $A \sim B$ exactly when $d_{\infty}(A, B)=\|A+B\|_{\infty}=2$. Then referring to the second paragraph of CASE I., we deduce that $A \sim B$ if and only if one of the numbers $-1,1$ is an eigenvalue for $A$, both of them are eigenvalues for $B$ and the corresponding eigenspaces have two nontrivial intersections, or reversely. This characterization easily implies the next equivalence. The condition that there is no operator $Y \in \mathcal{S}_{0}(\mathcal{H})_{1}$ such that $A \sim Y$ is necessary and sufficient for $A$ to satisfy $(*)$.

From now on, we assume only that $n>2$ concerning $n$. Summing up what have been proven in the last two paragraphs, we conclude that $\phi$ preserves the elements $A$ of $\mathcal{S}_{0}(\mathcal{H})_{1}$ with the property $(*)$. It is clear that $\left(^{*}\right)$ holds for $A$ exactly when

$$
A=\tau \oplus A^{\prime},
$$


where $\tau \in\{-1,1\}$ is a real number and $A^{\prime}$ is a self-adjoint operator on an $n$-1-dimensional subspace of $\mathcal{H}$ with $\operatorname{tr} A^{\prime}=-\tau,\left\|A^{\prime}\right\|_{\infty}<1$ (in the paper $\oplus$ stands for the direct sum of Hilbert space operators). We denote the set of all operators of this form by $\mathcal{E}(\mathcal{H})$. We emphasize the plain fact that for any element $A \in \mathcal{E}(\mathcal{H})$ the last displayed decomposition is unique. Now it is also clear that $\phi(\mathcal{E}(\mathcal{H})) \subset \mathcal{E}(\mathcal{H})$. Let $A, B \in \mathcal{E}(\mathcal{H})$ be elements of the forms $A=\tau \oplus A^{\prime}, B=\tau \oplus B^{\prime}$, where $\tau \in\{-1,1\}$ is a real number and $A^{\prime}, B^{\prime}$ are operators on a subspace $\mathcal{M}$ of $\mathcal{H}$ with $\operatorname{dim} \mathcal{M}=n-1$. Then $\|A+B\|_{\infty}=2$ and therefore for the operators $\phi(A), \phi(B) \in \mathcal{E}(\mathcal{H})$ we have $\|\phi(A)+\phi(B)\|=2$. By the end of the second paragraph of CASE I., now we get that $\phi(A)=\tau^{\prime} \oplus A^{\prime \prime}, \phi(B)=\tau^{\prime} \oplus B^{\prime \prime}$ with some number $\tau^{\prime} \in\{-1,1\}$ and with certain operators $A^{\prime \prime}, B^{\prime \prime}$ on an $n$-1-dimensional subspace $\mathcal{M}^{\prime} \subset \mathcal{H}$.

In what follows let $\mathcal{L}$ be a fixed subspace of $\mathcal{H}$ of codimension 1 and denote by $\mathcal{G}(\mathcal{L})$ the set of those elements $A \in \mathcal{S}(\mathcal{L})$ which satisfy that $\operatorname{tr} A=-1,\|A\|_{\infty}<1$. Then by the previous paragraph, there exists a number $\tau_{\mathcal{L}} \in\{-1,1\}$ and a subspace $\mathcal{L}^{\prime} \subset \mathcal{H}$ with $\operatorname{dim} \mathcal{L}^{\prime}=n-1$ such that for any $A \in \mathcal{G}(\mathcal{L})$ we have an operator $A^{\prime}$ on $\mathcal{L}^{\prime}$ for which

$$
\phi(1 \oplus A)=\tau_{\mathcal{L}} \oplus A^{\prime} \text { and } \tau_{\mathcal{L}} A^{\prime} \in \mathcal{G}\left(\mathcal{L}^{\prime}\right) .
$$

We define a transformation $\phi_{\mathcal{L}}: \mathcal{G}(\mathcal{L}) \rightarrow \mathcal{G}\left(\mathcal{L}^{\prime}\right)$ by $\phi_{\mathcal{L}}(A)=\tau_{\mathcal{L}} A^{\prime}(A \in \mathcal{G}(\mathcal{L}))$. Then using the previous paragraph it is easy to check that $\phi_{\mathcal{L}}$ is a well-defined isometry with $\operatorname{rng} \phi_{\mathcal{L}}=\mathcal{G}\left(\mathcal{L}^{\prime}\right)$. Since $\operatorname{dim} \mathcal{L}=\operatorname{dim} \mathcal{L}^{\prime}$, there exists a unitary operator $V_{\mathcal{L}}$ from $\mathcal{L}$ onto $\mathcal{L}^{\prime}$. Let $\mathcal{I}(\mathcal{L})$ be the set of those elements $A \in \mathcal{S}_{0}(\mathcal{L})$ for which $\left.\sigma(A) \subset\right]-1+1 /(n-1), 1+1 /(n-1)[$. Now consider the map $\Phi_{\mathcal{L}}: \mathcal{I}(\mathcal{L}) \rightarrow \mathcal{I}(\mathcal{L})$ defined by

$$
\Phi_{\mathcal{L}}(A)=V_{\mathcal{L}}^{-1} \phi_{\mathcal{L}}\left(A+\frac{1}{1-n} I\right) V_{\mathcal{L}}+\frac{1}{n-1} I \quad(A \in \mathcal{I}(\mathcal{L}))
$$

Elementary considerations show that $\Phi_{\mathcal{L}}$ is a surjective isometry of $\mathcal{I}(\mathcal{L})$. Moreover one can check that $\mathcal{I}(\mathcal{L})$ is an open convex subset of $\mathcal{S}_{0}(\mathcal{L})$. A result of Mankiewicz [10, Theorem 5] tells us that any surjective isometry between convex subsets of normed linear spaces with nonempty interiors can be extended to a bijective affine isometry between the ambient spaces. Applying this statement we obtain that $\Phi_{\mathcal{L}}$ possesses such an extension which is a selfmap of $\mathcal{S}_{0}(\mathcal{L})$. By the inductive hypothesis the latter map is of the form $A \mapsto \mu_{\mathcal{L}} U_{\mathcal{L}} A U_{\mathcal{L}}^{*}+X_{\mathcal{L}}\left(A \in \mathcal{S}_{0}(\mathcal{L})\right)$ with some scalar $\mu_{\mathcal{L}} \in\{-1,1\}$, operator $X \in \mathcal{S}_{0}(\mathcal{L})$ and unitary or antiunitary operator $U$ on $\mathcal{L}$. Therefore $\Phi_{\mathcal{L}}$ can also be written in this form and by the fact that $\operatorname{rng} \Phi_{\mathcal{L}} \subset \mathcal{I}(\mathcal{L})$, we easily infer $X=0$. Now putting together all the information we have, we arrive at the conclusion that up to a unitary or antiunitary similarity transformation, $\phi_{\mathcal{L}}$ is the map $A \mapsto \tau_{\mathcal{L}} A(A \in \mathcal{G}(\mathcal{L}))$ which implies

$$
\phi\left(1 \oplus \frac{1}{1-n} I_{\mathcal{L}}\right) \in\left\{1 \oplus \frac{1}{1-n} I_{\mathcal{L}^{\prime}},-\left(1 \oplus \frac{1}{1-n} I_{\mathcal{L}^{\prime}}\right)\right\} .
$$

Let $P \in \mathcal{P}_{1}(\mathcal{H})$ be an operator. Referring to the last displayed formula, it is very easy to see that there is an element $P^{\prime} \in \mathcal{P}_{1}(\mathcal{H})$ such that $\phi(P+(1 /(1-n))(I-P))$ is $P^{\prime}+(1 /(1-n))\left(I-P^{\prime}\right)$ or $-\left(P^{\prime}+(1 /(1-n))\left(I-P^{\prime}\right)\right)$. The linearity of $\phi$ implies that

$$
\phi(n P-I) \in\left\{n P^{\prime}-I,-\left(n P^{\prime}-I\right)\right\}
$$

and therefore $\sigma(\phi(n P-I))$ is one of two different sets, $\{-1, n-1\}$ or $\{1-n, 1\}$. Then referring to the connectedness of $\mathcal{P}_{1}(\mathcal{H})$ and to the continuity of $\phi$ and the spectrum, we deduce that $\sigma(\phi(n P-I))=\{-1, n-1\}$ for all $P \in \mathcal{P}_{1}(\mathcal{H})$ or $\sigma(\phi(n P-I))=\{1-n, 1\}$ for each $P \in \mathcal{P}_{1}(\mathcal{H})$. It follows that one has the following possibilities: 
(i) For any projection $P \in \mathcal{P}_{1}(\mathcal{H})$ there is a unique element $\psi(P) \in \mathcal{P}_{1}(\mathcal{H})$ such that

$$
\phi(n P-I)=n \psi(P)-I .
$$

(ii) For every operator $P \in \mathcal{P}_{1}(\mathcal{H})$ we have exactly one element $\psi(P) \in \mathcal{P}_{1}(\mathcal{H})$ satisfying

$$
\phi(n P-I)=-(n \psi(P)-I) .
$$

Since the inverse of the bijection $\phi: \mathcal{S}_{0}(\mathcal{H}) \rightarrow \mathcal{S}_{0}(\mathcal{H})$ has the same preserver properties as $\phi$, it is obvious that $\psi: \mathcal{P}_{1}(\mathcal{H}) \rightarrow \mathcal{P}_{1}(\mathcal{H})$ is a surjective isometry of $\mathcal{P}_{1}(\mathcal{H})$. Then, applying [3, Theorem 1.1.] we obtain the existence of a unitary or an antiunitary operator $U$ on $\mathcal{H}$ for which $\psi$ can be written in the form $\psi(P)=U P U^{*}\left(P \in \mathcal{P}_{1}(\mathcal{H})\right)$. By the definition of $\psi$ and the latter formula, there is a scalar $\mu \in\{-1,1\}$ such that

$$
\phi(n P-I)=\mu U(n P-I) U^{*} \quad\left(P \in \mathcal{P}_{1}(\mathcal{H})\right) .
$$

To complete the proof of the first assertion in Theorem let $A \in \mathcal{S}_{0}(\mathcal{H})$ be an operator. Then referring to the spectral theorem and to the property $\operatorname{tr} A=0$, we have

$$
A=\sum_{i=1}^{n} \lambda_{i} P_{i}
$$

for certain projections $P_{1}, \ldots, P_{n} \in \mathcal{P}_{1}(\mathcal{H})$ and for some real numbers $\lambda_{1}, \ldots, \lambda_{n}$ which sum up to 0 . We compute

$$
A=\sum_{i=1}^{n} \frac{\lambda_{i}}{n}\left(n P_{i}-I\right)
$$

and this, by the formula (2) and by the linearity of $\phi$ yields $\phi(A)=\mu U A U^{*}$. Since $A$ was an arbitrary element of $\mathcal{S}_{0}(\mathcal{H})$, now we obtain the first statement in Theorem for $n$-dimensional complex Hilbert spaces and then its proof is complete.

CASE II. In the rest of this section we suppose that $p<\infty$ and $\operatorname{dim} \mathcal{H} \neq 3$. We will need the definition of orthogonality of elements in $\mathcal{S}(\mathcal{H})$ which reads as follows. The operators $A, B \in \mathcal{S}(\mathcal{H})$ are orthogonal if $A B=0$ which is equivalent to the condition that they have mutually orthogonal ranges. We say that $\phi$ preserves orthogonality if for any $A, B \in \mathcal{S}_{0}(\mathcal{H})$ one has $\phi(A) \phi(B)=0$ exactly when $A B=0$. We remark that in the case $\operatorname{dim} \mathcal{H}=3$, there are no pairs of orthogonal nonzero elements in $\mathcal{S}_{0}(\mathcal{H})$. So in the latter case, the orthogonality preserving property of $\phi$ holds if and only if for any $A \in \mathcal{S}_{0}(\mathcal{H})$ one has $A=0$ if and only if $\phi(A)=0$. The first step of the argument below is to show that $\phi$ has this property and using it, in the second one we are going to verify that $\phi$ leaves the set of rank two elements of $\mathcal{S}_{0}(\mathcal{H})$ invariant. But - as we have seen above if $\operatorname{dim} \mathcal{H}=3$, then the orthogonality preserving property of $\phi$ gives us practically no information. That is the reason why we assume that $\operatorname{dim} \mathcal{H} \neq 3$ in the course of the proof.

We need the following result concerning the $p$-norm which can be found in, e.g. [11] as Theorem 2.7. For any linear operators $S, T$ on $\mathcal{H}$ one has

$$
\|S+T\|_{p}^{p}+\|S-T\|_{p}^{p}=2\left(\|S\|_{p}^{p}+\|T\|_{p}^{p}\right) \Longleftrightarrow S^{*} S T^{*} T=0 .
$$

Now let $A, B \in \mathcal{S}_{0}(\mathcal{H})$. Then we have

$$
A^{*} A B^{*} B=0 \Longleftrightarrow A B=0 .
$$


To see that $A^{*} A B^{*} B=0$ implies $A B=0$, observe that if the former equality holds, then $A^{2} B^{2}=0$ meaning that $A^{2}, B^{2}$ are orthogonal. It is very easy to check that the ranges of an element of $\mathcal{S}(\mathcal{H})$ and its square are the same and then it follows that $A, B$ are orthogonal, i.e. $A B=0$. Now using $(3),(4)$ and the linearity and isometric property of $\phi$, we conclude that it preserves orthogonality. Let $\mathcal{M} \subset \mathcal{S}_{0}(\mathcal{H})$ be a set and let us define

$$
\mathcal{M}^{\perp}=\left\{T \in \mathcal{S}_{0}(\mathcal{H}) \mid T M=0 \text { for all } M \in \mathcal{M}\right\} .
$$

Since the surjective map $\phi$ preserves zero products, we infer that $\phi\left(\mathcal{M}^{\perp}\right)=\phi(\mathcal{M})^{\perp}$. We can characterize the operators in $\mathcal{S}_{0}(\mathcal{H})$ of corank at least 2 as those elements $A \in \mathcal{S}_{0}(\mathcal{H})$ for which $\{A\}^{\perp}$ has a nonzero member. We deduce that $\phi$ preserves such operators in both directions. One can check that for each $A \in \mathcal{S}_{0}(\mathcal{H})$ with rank at most $n-2$, the equality

$$
\left(\{A\}^{\perp}\right)^{\perp}=\left\{T \in \mathcal{S}_{0}(\mathcal{H}) \mid \operatorname{rng} T \subset \operatorname{rng} A\right\}
$$

holds. Let $A, B \in \mathcal{S}_{0}(\mathcal{H})$ be operators of corank at least 2. It follows easily that if $\operatorname{rng} A \subset \operatorname{rng} B$, then $\operatorname{rng} \phi(A) \subset \operatorname{rng} \phi(B)$. This implies that in the case where $\operatorname{rng} A=\operatorname{rng} B$ one has $\operatorname{rng} \phi(A)=$ $\operatorname{rng} \phi(B)$ and now we deduce that if $\operatorname{rng} A \subsetneq \operatorname{rng} B$, then $\operatorname{rng} \phi(A) \subsetneq \operatorname{rng} \phi(B)$.

We assert that $\phi$ preserves the rank-two elements. Since it is injective, sends 0 to 0 and leaves the set of operators of rank at most $n-2$ invariant, this clearly holds in the case $n=2,4$. Accordingly, in what follows, we will assume that $n>4$. Let $A \in \mathcal{S}_{0}(\mathcal{H})$ be an operator of rank 2 . Then one can check that there are elements $A_{1}, \ldots, A_{n-4} \in \mathcal{S}_{0}(\mathcal{H})$ of corank at least 2 such that $\operatorname{rng} A \subsetneq \operatorname{rng} A_{1} \subsetneq \ldots \subsetneq \operatorname{rng} A_{n-4}$ which implies

$$
\operatorname{rng} \phi(A) \subsetneq \operatorname{rng} \phi\left(A_{1}\right) \subsetneq \ldots \subsetneq \operatorname{rng} \phi\left(A_{n-4}\right) .
$$

This gives us that the rank of $\phi(A)$ can not be greater than 2 , therefore it is 2 . Now we conclude that in both cases, $\phi$ preserves the set of operators of rank 2. Such elements of $\mathcal{S}_{0}(\mathcal{H})$ are exactly the operators of the form $a(P-Q)$, where $P, Q \in \mathcal{P}_{1}(\mathcal{H})$ are mutually orthogonal projections and $a$ is a nonzero real number. Referring to the fact that $\phi$ is clearly norm preserving, it then follows that $\phi$ leaves $\mathcal{D} \mathcal{P}_{1}(\mathcal{H})$ invariant (for the definition of $\mathcal{D} \mathcal{P}_{1}(\mathcal{H})$ see $(1)$ ).

Now we assert that $\phi$ preserves the quantity $\operatorname{diam} \sigma(A)\left(A \in \mathcal{S}_{0}(\mathcal{H})\right)$, i.e. the diameter of the spectrum. We divide the corresponding proof into two subcases.

Case II.a. Now we deal with the case $p=1$. Let $A \in \mathcal{S}_{0}(\mathcal{H})$ be an operator satisfying $\|A\|_{1}=1$. We are going to compute the infimum of $\left\{d_{1}(A, S) \mid S \in \mathcal{D P} \mathcal{P}_{1}(\mathcal{H})\right\}$. To this end, first we remark that as it is well-known, the Schatten norms are unitarily invariant meaning that the norm of a linear operator does not change under multiplication by a unitary operator. In what follows, for an element $N \in \mathcal{S}(\mathcal{H})$ and a number $i=1, \ldots, n$ we denote by $\lambda_{i}(N)$ the $i$ th smallest eigenvalue of $N$. Now let $N, N^{\prime}, R, R^{\prime} \in \mathcal{S}(\mathcal{H})$ be operators such that for some decomposition of $\mathcal{H}$ we have

$$
N^{\prime}=\lambda_{n}(N) \oplus \ldots \oplus \lambda_{1}(N), R^{\prime}=\lambda_{n}(R) \oplus \ldots \oplus \lambda_{1}(R) .
$$

We learn from [2, Theorem 9.7] that if $\|\cdot\|$ is a unitarily invariant norm on the space of linear operators on $\mathcal{H}$, then $\left\|N^{\prime}-R^{\prime}\right\| \leq\|N-R\|$. Now let $T \in \mathcal{D P}_{1}(\mathcal{H})$ be an arbitrary element. Then the previous discussion yields

$$
\sum_{i=1}^{n}\left|\lambda_{i}(A)-\lambda_{i}(T)\right| \leq\|A-T\|_{1},
$$


which gives us the inequality

$$
\left|\lambda_{n}(A)-1\right|+\sum_{i=2}^{n-1}\left|\lambda_{i}(A)\right|+\left|\lambda_{1}(A)+1\right| \leq\|A-T\|_{1} .
$$

As $\|A\|_{1}=1$ we have $-1 \leq \lambda_{1}(A)<0<\lambda_{n}(A) \leq 1$ and $\sum_{i=2}^{n-1}\left|\lambda_{i}(A)\right|=1-\left|\lambda_{1}(A)\right|-\left|\lambda_{n}(A)\right|$, therefore it follows that

$$
3+2\left(\lambda_{1}(A)-\lambda_{n}(A)\right) \leq\|A-T\|_{1}=d_{1}(A, T) .
$$

Now let $P_{M}$, resp. $P_{m}$ be the projection on $\mathcal{H}$ onto the subspace of $\mathcal{H}$ generated by an eigenvector of $A$ corresponding to $\lambda_{n}(A)$, resp. $\lambda_{1}(A)$. Then it is trivial that for the operator $T_{0}=P_{M}-P_{m} \in$ $\mathcal{D P} \mathcal{P}_{1}(\mathcal{H})$ one has $d_{1}\left(A, T_{0}\right)=3+2\left(\lambda_{1}(A)-\lambda_{n}(A)\right)$. Using the latter discussion we conclude that

$$
\inf \left\{d_{1}(A, S) \mid S \in \mathcal{D} \mathcal{P}_{1}(\mathcal{H})\right\}=3+2\left(\lambda_{1}(A)-\lambda_{n}(A)\right)=3-2 \operatorname{diam} \sigma(A)
$$

and as $\phi\left(\mathcal{D} \mathcal{P}_{1}(\mathcal{H})\right)=\mathcal{D} \mathcal{P}_{1}(\mathcal{H})$, now it follows easily that $\operatorname{diam} \sigma(\phi(A))=\operatorname{diam} \sigma(A)$. Since $A$ was an arbitrary normalized element of $\mathcal{S}_{0}(\mathcal{H})$, by the homogeneity of $\phi$ we then deduce that it preserves the diameter of the spectrum in the case $p=1$.

Case II.b. In the present subcase, we assume that $p \neq 1$. Let $A, B \in \mathcal{S}_{0}(\mathcal{H})$ be nonzero operators. Then, referring to [1, Theorem 2.3], the function $t \mapsto\|A+t B\|_{p}(t \in \mathbb{R})$ is differentiable at 0 and

$$
\left.\frac{\mathrm{d}\|A+t B\|_{p}}{\mathrm{~d} t}\right|_{t=0}=\frac{\operatorname{tr}|A|^{p-1} V_{A}^{*} B}{\|A\|_{p}^{p-1}},
$$

where $V_{A}$ is the partial isometry appearing in the polar decomposition of $A$, which is easily seen to be $\operatorname{sgn} A$. Let $f: \mathbb{R} \rightarrow \mathbb{R}$ be the map defined by $f(t)=\operatorname{sgn} t|t|^{p-1}(t \in \mathbb{R})$. We infer that

$$
\left.\frac{\mathrm{d}\|A+t B\|_{p}}{\mathrm{~d} t}\right|_{t=0}=\frac{\operatorname{tr} f(A) B}{\|A\|_{p}^{p-1}} .
$$

By the linearity and the norm preserving property of $\phi$ one has $\|A+t B\|_{p}=\|\phi(A)+t \phi(B)\|_{p}(t \in \mathbb{R})$ and now it follows easily that $\operatorname{tr} f(\phi(A)) \phi(B)=\operatorname{tr} f(A) B$. It is clear that $f(A)=A$ for any operator $A \in \mathcal{D P}_{1}(\mathcal{H})$ and then since $\phi\left(\mathcal{D P}_{1}(\mathcal{H})\right)=\mathcal{D P}_{1}(\mathcal{H})$, the previous discussion yields that for each nonzero element $A \in \mathcal{S}_{0}(\mathcal{H})$

$$
\sup \left\{\operatorname{tr} \phi(A) T \mid T \in \mathcal{D} \mathcal{P}_{1}(\mathcal{H})\right\}=\sup \left\{\operatorname{tr} A T \mid T \in \mathcal{D} \mathcal{P}_{1}(\mathcal{H})\right\}
$$

Let $A$ be such an operator and $T$ be an element of $\mathcal{D} \mathcal{P}_{1}(\mathcal{H})$. There are mutually orthogonal unit vectors $x, y \in \mathcal{H}$ such that $T=x \otimes x-y \otimes y$ (in this paper $\otimes$ denotes the tensor product of vectors of $\mathcal{H}$ in the sense of functional analysis). We then compute

$$
\operatorname{tr} A T=\operatorname{tr} A x \otimes x-\operatorname{tr} A y \otimes y=\langle A x, x\rangle-\langle A y, y\rangle,
$$

and thus, using the fact that the numerical range of the self-adjoint operator $A$ lies in $[\min \sigma(A), \max \sigma(A)]$ we infer that $\operatorname{tr} A T$ is majorized by the diameter $\operatorname{diam} \sigma(A)$ of $\sigma(A)$, i.e. $\operatorname{tr} A T \leq \operatorname{diam} \sigma(A)$. Let $e_{m}$, resp. $e_{M}$ be a normalized eigenvector of $A$ corresponding to $\min \sigma(A)$, resp. $\max \sigma(A)$. Then for the operator

$$
T_{0}=e_{M} \otimes e_{M}-e_{m} \otimes e_{m} \in \mathcal{D} \mathcal{P}_{1}(\mathcal{H})
$$


we have $\operatorname{tr} A T_{0}=\operatorname{diam} \sigma(A)$ and hence the previous argument gives us that

$$
\sup \left\{\operatorname{tr} A T \mid T \in \mathcal{D} \mathcal{P}_{1}(\mathcal{H})\right\}=\operatorname{diam} \sigma(A) .
$$

Referring to (5), we now conclude that

$$
\operatorname{diam} \sigma(\phi(A))=\operatorname{diam} \sigma(A) \quad\left(A \in \mathcal{S}_{0}(\mathcal{H})\right)
$$

and therefore we get that $\phi$ preserves the diameter of the spectrum in the case $p \neq 1$.

In the rest of this section, the only condition which we postulate concerning $p$ is that $1 \leq p \neq 2$. Let $\Phi: \mathcal{S}(\mathcal{H}) \rightarrow \mathcal{S}(\mathcal{H})$ be the unique linear extension of $\phi$ onto $\mathcal{S}(\mathcal{H})$ with the property $\Phi(I)=I$. Referring to Cases II.a. and II.b., we see that (6) holds true and therefore, by the bijectivity of $\phi$ we get that $\Phi$ is a linear automorphism of $\mathcal{S}(\mathcal{H})$ which preserves the diameter of the spectrum. To see this property, observe that $\operatorname{diam} \sigma(A)=\operatorname{diam} \sigma(A+\alpha I)$ for any $A \in \mathcal{S}(\mathcal{H})$ and $\alpha \in \mathbb{R}$. Now using (6), the linearity of $\Phi$ and the equality $\Phi(I)=I$, it follows that $\Phi$ preserves the diameter of the spectrum. The result [12, Theorem 2] describes the structure of those linear bijections on the space of all self-adjoint operators on a Hilbert space which leave the so-called maximal deviation invariant. According to [12, Lemma 1], this quantity is the half of the diameter of the spectrum, therefore the former statement tells us the general form of those linear automorphisms of $\mathcal{S}(\mathcal{H})$ which preserve the quantity $\operatorname{diam} \sigma(A)(A \in \mathcal{S}(\mathcal{H}))$. Applying [12, Theorem 2] to $\Phi$ we obtain that there exist a unitary or an antiunitary operator $U$ on $\mathcal{H}$, a linear functional $\Lambda: \mathcal{S}(\mathcal{H}) \rightarrow \mathbb{R}$ and a number $\tau \in\{-1,1\}$ such that $\Phi$ can be written in the form

$$
\Phi(A)=\tau U A U^{*}+\Lambda(A) I \quad(A \in \mathcal{S}(\mathcal{H})) .
$$

Then using the fact that $\phi$ is a restriction of $\Phi$, we get that $\phi$ is of one of the forms appearing in Theorem and now we are done.

Remark. A natural question arises concerning Theorem. Namely, what can be said about the isometries of $\mathcal{S}_{0}(\mathcal{H})$ in the "missing" cases. In fact, Theorem does not contain any statement about those maps in the case where the underlying metric is $d_{2}$, and it does not cover the case $\operatorname{dim} \mathcal{H}=3$ when $d_{p}(1 \leq p<\infty)$ is considered. As for the isometries of $\mathcal{S}_{0}(\mathcal{H})$ under $d_{2}$, since this distance comes from an inner product, there are an abundance of them, they have no structure. We conjecture that in the case where $1 \leq p<\infty$ and $\operatorname{dim} \mathcal{H}=3$, the isometries of $\left(\mathcal{S}_{0}(\mathcal{H}), d_{p}\right)$ are of one of the forms appearing in Theorem. As for possible proofs, it is clear that one has to apply an argument which is much different from the one in CASE II. Theorem gives the structure of isometries of $\mathcal{S}_{0}(\mathcal{H})$ relative to metrics induced by a well-known class of unitarily invariant norms. As for other norms of that kind, applying an argument similar to that in the first three paragraphs of the proof of Theorem, one can show that in the case $\operatorname{dim} \mathcal{H}=2$ the corresponding isometries are of one of the forms appearing in that statement. However, in higher dimensions, we do not know anything about their structure. We propose the problem of determining their general form as an open question for future research.

Acknowledgements. The author was supported by the "Lendület" Program (LP2012-46/2012) of the Hungarian Academy of Sciences. He would like to express his gratitude to the referee for the useful comments. He is grateful to Prof. L. Molnár for his kind advices that helped improving the presentation of the paper. 
[1] T. J. Abatzoglou, Norm derivatives on spaces of operators, Math. Ann. 239 (1979), 129-135.

[2] R. Bhatia, Perturbation bounds for matrix eigenvalues, Longman, Essex and Wiley, New York, 1987.

[3] F. Botelho, J. Jamison and L. Molnár, Surjective isometries on Grassmann spaces, J. Funct. Anal. 265 (2013), $2226-2238$.

[4] R. C. Bourgin, Approximate isometries on finite dimensional Banach spaces, Trans. Amer. Math. Soc. 207 (1975), 309-328.

[5] R. J. Fleming and J. E. Jamison, Isometries on Banach Spaces: Function Spaces, CRC Press, Boca Raton, FL, 2003.

[6] R. J. Fleming and J. E. Jamison, Isometries on Banach Spaces: Vector-valued Function Spaces and Operator Spaces, CRC Press, Boca Raton, FL, 2007.

[7] O. Hatori, Isometries on the special unitary group, to appear in a volume of the series "Contemporary Mathematics".

[8] R. V. Kadison, Isometries of operator algebras, Ann. Math. 54 (1951), 325-338.

[9] R. V. Kadison, A generalized Schwarz inequality and algebraic invariants for operator algebras, Ann. Math. 56 (1952), 494-503.

[10] P. Mankiewicz, On extension of isometries in normed linear spaces, Bull. Acad. Pol. Sci., Sér. Sci. Math. Astron. Phys. 20 (1972), 367-371.

[11] C. A. McCarthy, $c_{p}$, Israel J. Math. 5 (1967), 249-271.

[12] L. Molnár and M. Barczy, Linear maps on the space of all bounded observables preserving maximal deviation, J. Funct. Anal. 205 (2003), 380-400. 